\title{
Emission-line Galaxies in Shahbazian Compact Groups
}

\author{
H.Tiersch ${ }^{1,2}$ and D.Stoll ${ }^{1}$ \\ Sternwarte Koenigsleiten, München, Germany \\ S.Neizvestny \\ Special Astrophysical Observatory, Nizhny Arkhys, Russia
}

\author{
A.S.Amirkhanian and A.G. Egikian \\ Byurakan Astrophysical Observatory, Byurakan, 378433, Armenia
}

Based on the CCD photometric and spectroscopic observations taken with $1.23 \mathrm{~m}$ and $2.2 \mathrm{~m}$ telescopes at the German-Spanish Astronomical Center in Calar-Alto (Almeria, Spain) as well as with the $1.54 \mathrm{~m}$ telescope at the European Southern Observatory (La Silla, Chile) some emission-line galaxies (ELGs) surprisingly have been found in Shahbazian compact groups of galaxies (SHCGs) by our research group.

The majority of galaxies in the SHCGs usually have an absorption spectra typical of ellipticals and S0s. But some of these objects turn out to be the ELGs including two broad-line AGNs (of classical Seyfert 1 type) and the narrow emission-line galaxies. Their redshift makes these galaxies a physical member of the host group.

Unlike well studied Hickson compact groups (HCGs) (see e.g. Hickson 1993; Hickson 1997) not many SHCGs of galaxies have been investigated photometrically and spectroscopically till quite recently (Tiersch et al. 1998 and references therein). For this reason a program has been started in the University of Potsdam, Potsdam Astrophysikalisches Institut in cooperation with other observatories to obtain photometric and spectroscopic properties of the galaxies in SHCGs (Tiersch et al. 1994; Oleak et al. 1995; Tiersch et al. 1995).

The main goal of our program is to study the physical nature of these groups, their past and future evolutionary histories. With this purpose it is necessary to know the morphology of member galaxies, the photometric data, i. e. $B, V, R$ apparent magnitudes, radial velocities, velocity dispersion, mass-to-luminosity ratio, crossing times, dynamics, chemical abundance, metallicity as well as the evolution effects.

Three-colour $(B, V, R)$ photometric and spectroscopic observations have been carried out at Calar Alto and La Silla Observatories by Tiersch and Stoll. The data reduction procedure used the MIDAS software package at Potsdam Astrophysikalisches Institut.

\footnotetext{
${ }^{1}$ Visiting astronomer at the German-Spanish Astronomical Center, Calar Alto, Spain

${ }^{2}$ Visiting astronomer at the European Southern Observatory, La Silla,Chile
} 
The emission-line spectra are indicative of galaxies with active galactic nuclei (AGNs), star-forming galaxies which have had large bursts of star formation and normal late-type galaxies.

In the present study a total of 100 galaxies in 15 SHCGs has been spectroscopically investigated based on observations at Calar Alto. Eleven objects have emission-line spectra. One of them is a background AGN with the high - excitation ([OIII] $/ H_{\beta} \geq 3$ ) spectrum. This is galaxy number 7 in group $\mathrm{Sh}$ 354 ( Sh 354/7) - our first emission object with $z=0.1804 \pm 0.0001$. The mean redshift of the group has been found to be $z=0.0707$.

A total of 90 galaxies in 20 SHCGs has been spectroscopically observed at La Silla. Twelve objects exhibit emission lines. Two of them are foreground AGNs.

This sample is not at all complete. In order to complete, one must study all 377 groups in Shahbazian original lists (Shahbazian 1973; Baier and Tiersch 1979 and references therein).

According to these preliminary data about $10 \%$ of member galaxies in SHCGs are ELGs. This can be compared with the results obtained by Dressler, Thompson and Shectman (1985) for rich clusters of galaxies. Their statistics of emission - line galaxies are based on the spectra of 1268 galaxies in the fields of 14 rich clusters. About $7 \%$ of member galaxies in nearby $(z \sim 0.04)$ clusters have emission-line spectra.

A typical Shahbazian compact group is a relatively isolated association of five to fifteen galaxies within a small sky area of a few arcminutes across (Shahbazian 1973). The resulting spectra of the member galaxies usually show characteristics of K-type stars with absorption features of the $H_{\beta}, \mathrm{MgIb}, \mathrm{NaID}$ and $H_{\alpha}$ lines, which were used for the determination of the redshift. Reproduction of the absorption spectrum of the galaxy Sh 344/4 is shown in Figure 1. It is representative of an $\mathrm{E}$ type galaxy. The radial velocities were determined for five galaxies in the group Sh 344 from eight in the original list. All except one are group members with absorption spectra. One object is a foreground galaxy.

The first emission-line object in SHCGs was discovered by Spanish astronomers del Olmo and Moles (1991) at Calar Alto Observatory using the 3.5 m telescope. It is galaxy number 4 in group $\mathrm{Sh} 278$. Sh $278 / 4$ is the only active object found by them among a total of 49 galaxies in 11 SHCGs spectroscopically investigated. This low luminosity active nucleus hosted in an early-type galaxy has a remarkable resemblance with QSO or QSO-like objects.

Our spectrum of Sh 278/4 (see Fig.1) taken with the $1.54 \mathrm{~m}$ telescope at La Silla includes the $H_{\alpha}$ region which is absent in the spectrum optained at Calar Alto. The spectrum of the galaxy shows typical characteristics of broad-line AGNs, i.e. broad hydrogen lines $H_{\gamma}, H_{\beta}, H_{\alpha}$ with a high velocity FWHM of $\sim 7000 \mathrm{~km} / \mathrm{s}$. There are also the narrow forbidden lines of [OIII] 4959,5007, [NII] 6548,6584, [SII] 6717,6731 and weaker line of [OII] $3727 . H_{\alpha}$ is contaminated by [NII] 6548,6584. We classified Sh $278 / 4$ as a Seyfert 1 type galaxy.

The second active object found in our research is the galaxy Sh 355/4 (Tiersch et al. 1996). It is a classical Seyfert 1 type galaxy with very broad emission Balmer lines $H_{\beta}$ and $H_{\alpha}$ (see Fig. 1). The FWHM of $250 \mathrm{~A}$ is determined by the undisturbed $H_{\beta}$ line and corresponds to a very high velocity of about $15000 \mathrm{~km} / \mathrm{s}$. The spectrum also presents the strong forbidden lines $\mathrm{N} 1, \mathrm{~N} 2$ 
[OIII] and stellar absorption features MgIb and NaID which are absent in the spectrum of Sh $278 / 4$.

All other emission-line objects are narrow emission-line galaxies, among those Sh 307/7, Sh 331/1 and Sh 331/4 (see Fig.1). In their spectrum the permitted hydrogen lines are narrow as well as the forbidden ones. There are two member ELGs (numbers 1 and 4) in group Sh 331. Note the strongest $H_{\alpha}$ in the spectrum of Sh 302/7 and Sh 331/1.

The ELGs discovered by our group in these and other SHCGs now investigated will be the subject of a later more detailed spectrophotometric study. It is very important to know what is the population of emission-line galaxies found in SHCGs. What is their nature ? Which is their activity class?

Extensive spectroscopic studies of distant clusters of galaxies performed by Dressler, Gunn and collaborators (1992) over one decade showed that in distant clusters with redshifts between 0.37 and $0.5522 \%$ of all galaxies exhibit emission lines and about $13 \%$ reveal post-starburst features, while in nearby ones there are only $7 \%$ and $0.3 \%$, respectively. This seems to confirm the "ButcherOemler effect" which is the first observational evidence for the coeval evolution of galaxies (Butcher and Oemler 1984). Although the Butcher - Oemler effect was regarded with a lot of skepticism 15 years ago, it is now well established that galaxies in high redshift $(z \sim 0.4)$ rich clusters show a population of galaxies that are significantly bluer than their lower redshift counterparts (Dressler et al. 1994; Fabricant et al. 1994; Thimm et al. 1994). The fraction of blue galaxies increases from $5 \%$ at $z<0.1$ to $20 \%$ at $z=0.4$ (Dressler et al. 1985) and to $80 \%$ at $z=0.9$ (Rakos and Schombert 1995).

The Butcher-Oemler effect is also observed in radio selected groups of galaxies (Zirbel 1994). At high redshifts $(z \sim 0.4)$ rich groups ( of average density comparable to Abell richness class 0 ) have bluer colours. The blue galaxy population in these high redshift rich groups can be divided into two classes: the bright blue galaxies $\left(M_{v}<-21.5\right)$ that have disappeared today and the fainter blue galaxies whose evolution is more gradual. The bright blue galaxies may be identified with the "classical" Butcher-Oemler galaxies, post-starbursting ellipticals with strong Balmer lines (named E+A galaxies) discovered by Dressler and Gunn $(1983,1992)$, while the fainter blue galaxies may be late type galaxies (Zirbel 1994). Recent Hubble Space Telescope data showed that many of these "bluer" galaxies reveal a disturbed and peculiar morphology (Dressler et al. 1994).

On the other hand, now it is an observational fact that at low redshifts $(z<0.4)$, i. e. in the local Universe the QSOs and Butcher-Oemler galaxies have disappeared in the rich clusters of galaxies. Why? At redshifts 0.4 to 0.6 radio-loud QSOs are found in clusters of moderate to low richness (Ellingson et al. 1991). At lower redshifts the QSOs usually live in small groups of galaxies with a high density (Hutchings et al. 1993).

The comparison of nearby and distant galaxy groups' emission-line population probably is the key in understanding the formation and strong evolution of their member galaxies.

Acknowledgments. This work is supported by a grant of the "Deutsche Forschungsgemeinschaft". 

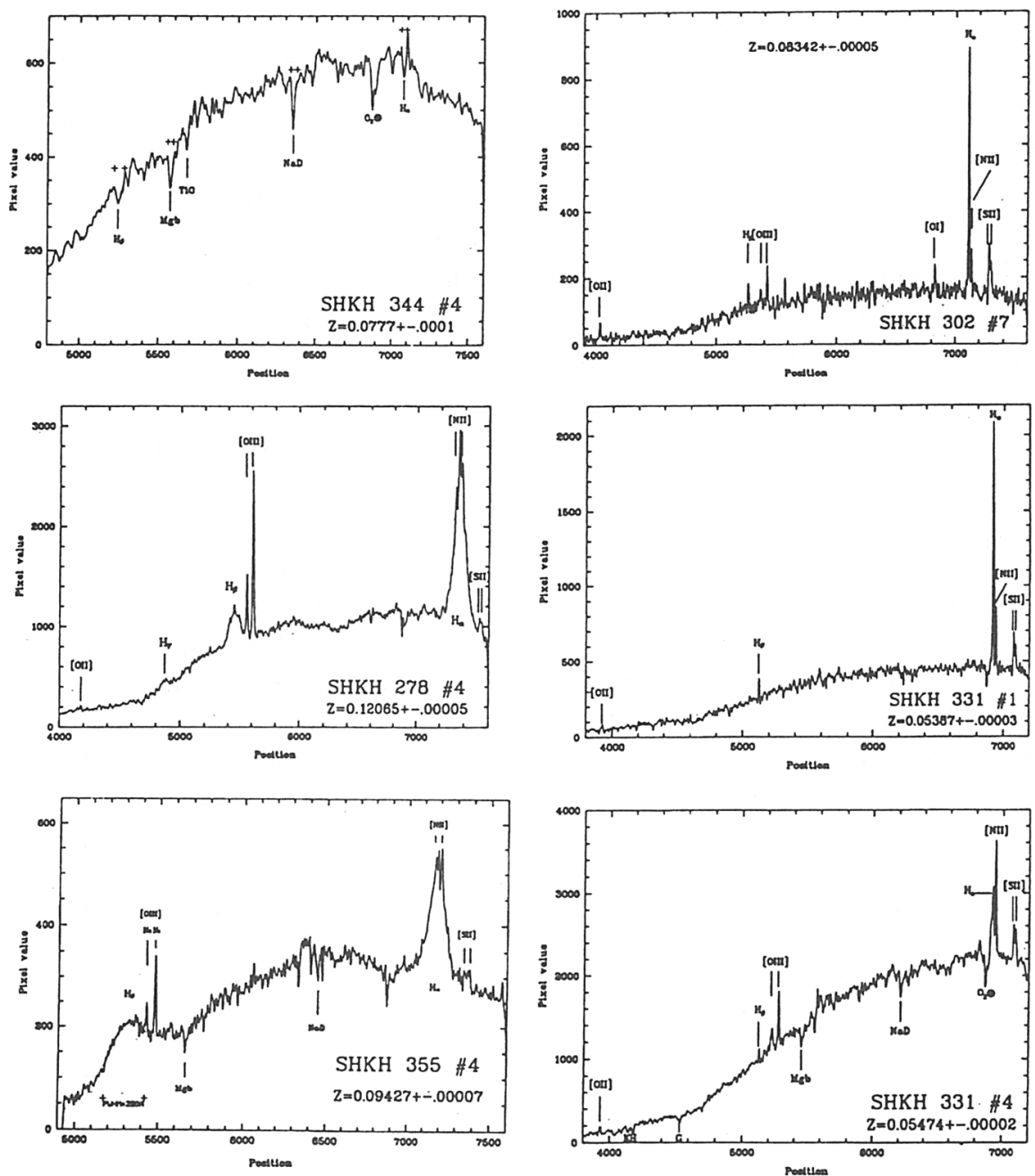

Figure 1. The absorption spectrum of Sh $344 / 4$ and the spectra of some ELGs in SHCGs. All except one were taken with the $1.5 \mathrm{~m}$ telescope at La Silla. The spectrum of Seyfert 1 galaxy Sh 355/4 was obtained using the $2.2 \mathrm{~m}$ telescope at Calar Alto. Original dispersion is $2.9 \stackrel{\AA}{A}$ per pixel, integration 3600 s. 


\section{References}

Baier, F.W., \& Tiersch, H. 1979, Astrofizika, 15, 33

Butcher, H., \& Oemler, A. 1984, ApJ, 285, 426

del Olmo, A., \& Moles, M. 1991, A\&A, 245, 27

Dressler, A., \& Gunn, J.E. 1983, ApJ, 270, 7

Dressler, A., Thompson, I.B., \& Shectman, S.A. 1985,ApJ., 288, 481

Dressler, A., Gunn, J.E., \& Schneider, D.P. 1985, ApJ, 294, 70

Dressler, A., \& Gunn, J.E. 1992, ApJS, 78, 1

Dressler, A.,Oemler, A., Sparks, W.B., \& Lucas, R.A. 1994, ApJ, 435, L23

Ellingson, E., Green, R.F., \& Yee, H.K.C. 1991, ApJ, 378, 476

Fabricant, D.G., Bautz, M.W., \& McClintock, J.E. 1994, AJ, 107, 8

Hickson, P. 1993, Astrophys.Lett.Commun., 29, Nos.1-3

Hickson, P. 1997, Ann. Rev. Astron. Astrophys., 35, 357

Hutchings, J.B., Crampton, D., \& Persram, D. 1993, DAO preprint

Oleak, H., Stoll, D., Tiersch, H., \& MacGillivray, H.T. 1995, AJ, 109, 1485

Racos, K., \& Schombert, J. 1995, ApJ, 439, 47

Shahbazian, R.K. 1973, Astrofizika, 9, 495

Thimm, G.J., Roser, H.-J., Hippelein, H., \& Meisenheimer, K. 1994, A\&A, 285, 785

Tiersch, H., Oleak, H., Stoll, D., \& Böhringer, H. 1994, in Astronomy from WideField Imaging, IAU Symp.161, ed. H.T.MacGillivray et al. (Dordrecht, Kluwer), 623

Tiersch, H., Oleak, H., Stoll, D., Neizvestny, S., Amirkhanian, A.S., \& Egikian, A.G. 1995, Astrofizika, 38, 688

Tiersch, H., Oleak, H., Stoll, D., Amirkhanian, A.S., \& Neizvestny, S. 1996, Astron. Soc. Pac. Conf. Ser., 98, 523

Tiersch, H., Stoll, D., Neizvestny, S., Amirkhanian, A.S., \& Egikian, A.G. 1998, in press

Zirbel, E.L. 1994, STSI preprint No. 856 